\title{
The Geographic Distribution of Excess Mortality Rate due to COVID-19 in Iranian Population: An Ecological Study
}

\author{
Alireza Molaei ${ }^{1}$ and Mohammad Gholami Fesharaki2 ${ }^{* *}$ \\ 1 Biostatistics MSC Student, Biostatistics Department, Faculty of Medical Sciences, Tarbiat Modares University, Tehran, Islamic Republic of Iran \\ 2 Associate Professor of Biostatistics, Biostatistics Department, Faculty of Medical Sciences, Tarbiat Modares University, Tehran, Islamic Republic of Iran \\ * Corresponding author: Mohammad Gholami Fesharaki, Department of Biostatistics, Faculty of Medical Sciences, Tarbiat Modares \\ University, Ale-Ahmad Highway, Tehran, Iran. Tel: +982182883578; Email: Mohammad.gholami@modares.ac.ir
}

Received 2020 August 10; Revised 2020 September 16; Accepted 2020 November 06.

\begin{abstract}
Background: COVID-19 has raised a worldwide trajectory since it emerged in Wuhan, China in December 2019. The direct and indirect mortalities in the world and as well as in Iran have increased significantly after the occurrence of this pandemic.

Objectives: In this study, Excess Mortality Rate (EMR) was estimated by multilevel poison regression method and then this estimation was compared to the historical trends to obtain total deaths related to COVID-19. Additionally, the geographic distribution of EMR has also been presented for Iran.

Methods: All-cause mortality rates of each province of Iran from March 21, 2013 to September 22, 2021 was downloaded from National Organization for Civil Registration (NOCR). The data of COVID-19 pandemic period (spring 1399 SH (Mar 20, 2020) to summer 1400 SH (Sep $22,2021)$ ) was removed from the data and then the multilevel poison model was applied to estimate all-cause mortality in this period. Then, $\mathrm{EMR}=$ (real deaths-expected death) $/$ (real deaths) ratio was calculated.

Results: The results of this study showed that Iran's EMR in COVID-19 pandemic was $36 \%$ (Male $=35 \%$, Female $=36 \%$, P-value $=0.798$ ). Our findings also revealed four category of EMR including low (EMR $\leq 30 \%, n=9)$, moderate $(30 \%<E M R \leq 35 \%, n=8)$, high $(35 \%<E M R \leq 40 \%$, $\mathrm{n}=10)$ and very high $(40 \%<\mathrm{EMR}, \mathrm{n}=4)$ in different provinces.

Conclusion: Due to the diverse EMR in different provinces of Iran, the type of disease management of provinces with low and moderate EMR can be used as an appropriate model to control EMR in provinces with high and very high EMR.
\end{abstract}

Keywords: Coronavirus, COVID-19, Excess deaths, Excess mortality, Iran, Mortality, SARS-CoV-2

\section{Background}

In late 2019, a wave of and acute respiratory diseases began in Wuhan (China). On March 11, 2020, according to the World Health Organization, the COVID-19, a pandemic was announced (1). The virus has been spreading from December 31, 2019 to September 22, 2021 around the world as it has infected 230,378,562 people and, to this day, has caused the death of more than 4,724,111 mortalities worldwide (2).

The number of diagnosed cases of COVID-19 in each country depends on the actual number of cases, the capacity of the health system to detect the cases, and its transparency in reporting the information. The health system's capacity to diagnose cases is dependent to multiple factors. The factors such as affordability and accessibility of healthcare facilities and diagnostic (screening tests), the accuracy of laboratory tests, public awareness, and the development of a mechanism for easy access to care facilities (3-5). The challenge is that the number of confirmed diagnosed cases does not reflect the actual number of cases (3).

In fact, due to various reasons such as the small number of diagnostic laboratories, lack of access to appropriate diagnostic kits and, health services due to excessive visits to medical centers, and high cost and low sensitivity of COVID-19 diagnostic tests, the real infection of COVID-19 of a majority of cases has not necessarily been reported by the Ministry of Health and Medical Education (MoHME) in routine statistical reports $(3,6)$. Therefore, estimating Excess Mortality Rate (EMR) from the National Organization for Civil Registration (NOCR) $(7,8)$ can be used as a tool for an estimation of COVID-19-related mortalities. This accurate estimation would enable the health managers to handle the epidemic better and more precisely.

\section{Objectives}

In this study, EMR was estimated by multilevel poison regression and then this estimation was compared to the historical trends to obtain total mortality rate attributable to COVID-19. Additionally, the geographic distribution of EMR in Iran was presented.

\section{Methods}

This study is a longitudinal ecological study. Allcause mortality rate of all provinces of Iran from March 21, 2013 to September 22, 2021 was downloaded from Iranian National Organization for Civil Registration (INOCR). The data from spring 
1399 SH (Mar 20, 2020) to summer 1400 SH (Sep $22,2021)$ was removed from the data pool and then the multilevel poison model was used to estimate all-cause mortality in the mentioned period (called expected death). After calculating the expected death, the EMR was calculated using the below formula:

$$
\mathrm{EMR}=\left(\frac{\text { real deaths }- \text { expected death }}{\text { real deaths }}\right)
$$

For the comparison of the rates reported by MoHME, the statistics reported by Islamic Republic News Agency (IRNA.ir) was used.

\subsection{Data Analysis}

In this study, due to the dependence of observations, it was not possible to use the Poisson model. Therefore, to model and estimate the number of deaths in the absence of COVID-19 pandemic, the multilevel Poisson model was applied. Lower type one error and higher efficiency are two advantages of multilevel models in comparison with conventional models in the comparison of correlated data (9).

Subsequently, the multilevel Poisson regression model was applied to predict the number of all-cause mortalities in COVID-19 pandemic, if this pandemic was not really occurred.

For this purpose, the data of the death of each province of Iran in the study time period (March 21, 2013 to Sep 22, 2021) was downloaded from the INOCR website (https://www.sabteahval.ir) and then the multilevel Poisson model (10) was run and to estimate the number of mortalities.

$$
\begin{aligned}
& \text { Death } h_{\text {its }} \sim \text { Poisson }\left(\mu_{\text {Death }} \text { its }\right) \\
& \operatorname{Ln}\left(\mu_{\text {Death }} \text { its }\right)=\beta_{0}+\beta_{1}\left(\text { Year }_{\text {its }}-1391\right)+\text { Season }^{\text {its }}+\text { Province }^{\text {its }}+u_{i} \\
& u_{i} \sim N\left(0, \sigma_{u}^{2}\right)
\end{aligned}
$$

Where index i varies from 1 to 31 number of provinces of Iran), index t varies from 1 to 8 (2013 to 2021) and index s ranges from 1 to 4 (4 seasons; spring, summer, autumn, and winter).

In the above model, $u_{-} \mathrm{i}$ is a normal random effect to control the dependence among observations. In the current study, for model estimation, Quasi-Likelihood (PQL) and Marginal Quasi-Likelihood (MQL) (11) were used. Moreover, MLwiN (version 2.1) and SPSS (version 2.1) were used to analyze the data. Finally, the geographical distribution of the EMR maps were prepared by online application of Paintmaps(12).

\subsection{Ethical considerations}

Ethical approval was not needed for this study, as it was based on surveillance data only and the confidential information of cases was not used or disclosed.

\section{Results}

According to the rates reported by the INOCR until summer 1400 SH (Sep 22, 2021), 797,755 deaths was reported. After fitting the multilevel Poisson model (R2=99\%), the model estimated 587,614 deaths in absence of COVID-19 pandemic. This is while according to the MoHME statistics, 117,905 deaths were found to be relevant to the pandemic. This means that the mortality rates related to the pandemic would be 1.78 times more than the statistics informed by MoHME .

The results of this study also demonstrated that EMR of Iran in COVID-19 pandemic was 36\% (Male $=35 \%$, Female $=36 \%$, P-value $=0.798$ ).

In Table 1 , the real and expected mortalities and EMR according to gender in the provinces of Iran is reported. As presented in this table the EMR of Iran in COVID-19 pandemic was 36\% (Male $=35 \%$, Female $=36 \%$, P-value $=0.798$ ). Our findings also revealed four categories of EMR which included low (EMR $\leq 30 \%, \mathrm{n}=9$, including provinces of Sistan \& Baluchestan, Chahar Mahall \& Bakhtiari, Gilan, South Khorasan, North Khorasan, Hormozgan, Kermanshah, Kohgiluyeh \& BuyerAhmad and Mazandaran), moderate $\quad(30 \%<\mathrm{EMR} \leq 35 \%, \mathrm{n}=8$, including provinces of Kerman, Lorestan, Markazi, Ilam, Fars, Semnan, Hamadan, Yazd ), high (35 \%< EMR $\leq 40 \%$, $\mathrm{n}=10$, including provinces of Bushehr, Qom, Kordestan, Razavi Khorasan, Zanjan, East Azarbaijan, Khuzestan, Golestan, Ardebil and Qazvin) and very high ( $40 \%<$ EMR, $n=4$, including provinces of West Azarbaijan, Esfahan, Tehran and Alborz ) .

The GIS map for EMR in addition to the mortality trend with and without the COVID-19 pandemic is presented in Figure 1 and Figure 2, respectively. In Figure 2, the trend line shows that the worst and the best pandemic controls were in the summer of 1400

\begin{tabular}{|c|c|c|c|c|c|c|c|c|c|}
\hline \multirow[b]{2}{*}{ Province } & \multicolumn{3}{|c|}{ Male } & \multicolumn{3}{|c|}{ Female } & \multicolumn{3}{|c|}{ Total } \\
\hline & $\begin{array}{c}\text { Expected } \\
\text { Death }\end{array}$ & $\begin{array}{c}\text { Real } \\
\text { Death }\end{array}$ & EMR & $\begin{array}{l}\text { Expected } \\
\text { Death }\end{array}$ & $\begin{array}{c}\text { Real } \\
\text { Death }\end{array}$ & EMR & $\begin{array}{l}\text { Expected } \\
\text { Death }\end{array}$ & $\begin{array}{c}\text { Real } \\
\text { Death }\end{array}$ & EMR \\
\hline East Azarbaijan & 19597 & 26406 & $35 \%$ & 15227 & 21620 & $42 \%$ & 34823 & 48026 & $38 \%$ \\
\hline West Azarbaijan & 13040 & 17621 & $35 \%$ & 10132 & 14939 & $47 \%$ & 23171 & 32560 & $41 \%$ \\
\hline Ardebil & 5889 & 7977 & $35 \%$ & 4576 & 6532 & $43 \%$ & 10465 & 14509 & $39 \%$ \\
\hline Esfahan & 20684 & 29374 & $42 \%$ & 16072 & 22634 & $41 \%$ & 36756 & 52008 & $41 \%$ \\
\hline
\end{tabular}
and then the winter of 1399 , respectively.

Table 1. The real and expected death and EMR according to gender and provinces of Iran in spring 1399 SH (Mar 20, 2020) to summer 1400 SH (Sep 22, 2021). 
Molaei A and Fesharaki MG.

\begin{tabular}{|c|c|c|c|c|c|c|c|c|c|}
\hline Alborz & 8859 & 15134 & $71 \%$ & 6883 & 11259 & $64 \%$ & 15742 & 26393 & $68 \%$ \\
\hline Ilam & 2176 & 3055 & $40 \%$ & 1690 & 2049 & $21 \%$ & 3866 & 5104 & $32 \%$ \\
\hline Bushehr & 3694 & 4995 & $35 \%$ & 2870 & 3943 & $37 \%$ & 6565 & 8938 & $36 \%$ \\
\hline Tehran & 50607 & 74517 & $47 \%$ & 39322 & 55676 & $42 \%$ & 89928 & 130193 & $45 \%$ \\
\hline ChaharMahall\&Bakhtiari & 3977 & 4698 & $18 \%$ & 3090 & 3556 & $15 \%$ & 7067 & 8254 & $17 \%$ \\
\hline South Khorasan & 3372 & 3973 & $18 \%$ & 2620 & 3228 & $23 \%$ & 5992 & 7201 & $20 \%$ \\
\hline Razavi Khorasan & 26872 & 36108 & $34 \%$ & 20880 & 29107 & $39 \%$ & 47752 & 65215 & $37 \%$ \\
\hline NorthKhorasan & 4013 & 4921 & $23 \%$ & 3118 & 4232 & $36 \%$ & 7132 & 9153 & $28 \%$ \\
\hline Khuzestan & 18077 & 25017 & $38 \%$ & 14046 & 19288 & $37 \%$ & 32123 & 44305 & $38 \%$ \\
\hline Zanjan & 4570 & 6079 & $33 \%$ & 3551 & 5011 & $41 \%$ & 8120 & 11090 & $37 \%$ \\
\hline Semnan & 2975 & 3946 & $33 \%$ & 2311 & 3075 & $33 \%$ & 5286 & 7021 & $33 \%$ \\
\hline Sistan\&Baluchestan & 11585 & 13779 & $19 \%$ & 9002 & 10129 & $13 \%$ & 20587 & 23908 & $16 \%$ \\
\hline Fars & 19195 & 25716 & $34 \%$ & 14914 & 19242 & $29 \%$ & 34109 & 44958 & $32 \%$ \\
\hline Qazvin & 5298 & 7263 & $37 \%$ & 4117 & 5958 & $45 \%$ & 9415 & 13221 & $40 \%$ \\
\hline Qom & 5343 & 7214 & $35 \%$ & 4151 & 5722 & $38 \%$ & 9494 & 12936 & $36 \%$ \\
\hline Kordestan & 6451 & 9011 & $40 \%$ & 5012 & 6603 & $32 \%$ & 11463 & 15614 & $36 \%$ \\
\hline Kerman & 11742 & 15313 & $30 \%$ & 9124 & 12016 & $32 \%$ & 20866 & 27329 & $31 \%$ \\
\hline Kermanshah & 9598 & 12847 & $34 \%$ & 7458 & 9293 & $25 \%$ & 17056 & 22140 & $30 \%$ \\
\hline Kohgiluyeh\&BuyerAhmad & 2406 & 3204 & $33 \%$ & 1869 & 2358 & $26 \%$ & 4275 & 5562 & $30 \%$ \\
\hline Golestan & 7779 & 10305 & $32 \%$ & 6044 & 8812 & $46 \%$ & 13823 & 19117 & $38 \%$ \\
\hline Gilan & 14817 & 16789 & $13 \%$ & 11513 & 14247 & $24 \%$ & 26330 & 31036 & $18 \%$ \\
\hline Lorestan & 7648 & 10250 & $34 \%$ & 5943 & 7596 & $28 \%$ & 13591 & 17846 & $31 \%$ \\
\hline Mazandaran & 14666 & 18241 & $24 \%$ & 11395 & 15697 & $38 \%$ & 26061 & 33938 & $30 \%$ \\
\hline Markazi & 6870 & 8880 & $29 \%$ & 5338 & 7118 & $33 \%$ & 12208 & 15998 & $31 \%$ \\
\hline Hormozgan & 5645 & 7608 & $35 \%$ & 4386 & 5188 & $18 \%$ & 10031 & 12796 & $28 \%$ \\
\hline Hamadan & 9027 & 11899 & $32 \%$ & 7014 & 9390 & $34 \%$ & 16041 & 21289 & $33 \%$ \\
\hline Yazd & 4207 & 5579 & $33 \%$ & 3269 & 4518 & $38 \%$ & 7477 & 10097 & $35 \%$ \\
\hline Total & 330676 & 447719 & $35 \%$ & 256937 & 350036 & $36 \%$ & 587614 & 797755 & $36 \%$ \\
\hline
\end{tabular}

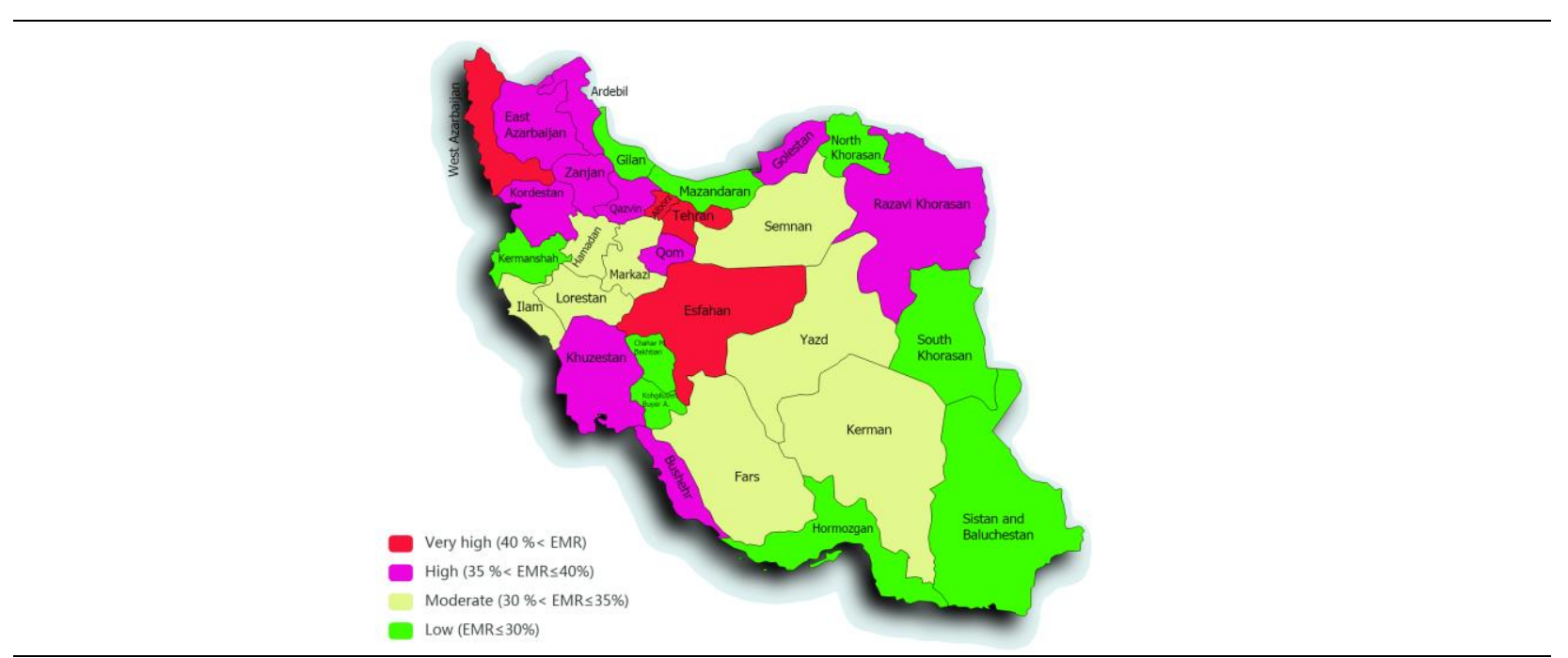

Figure 1. The GIS map for the rate of EMR

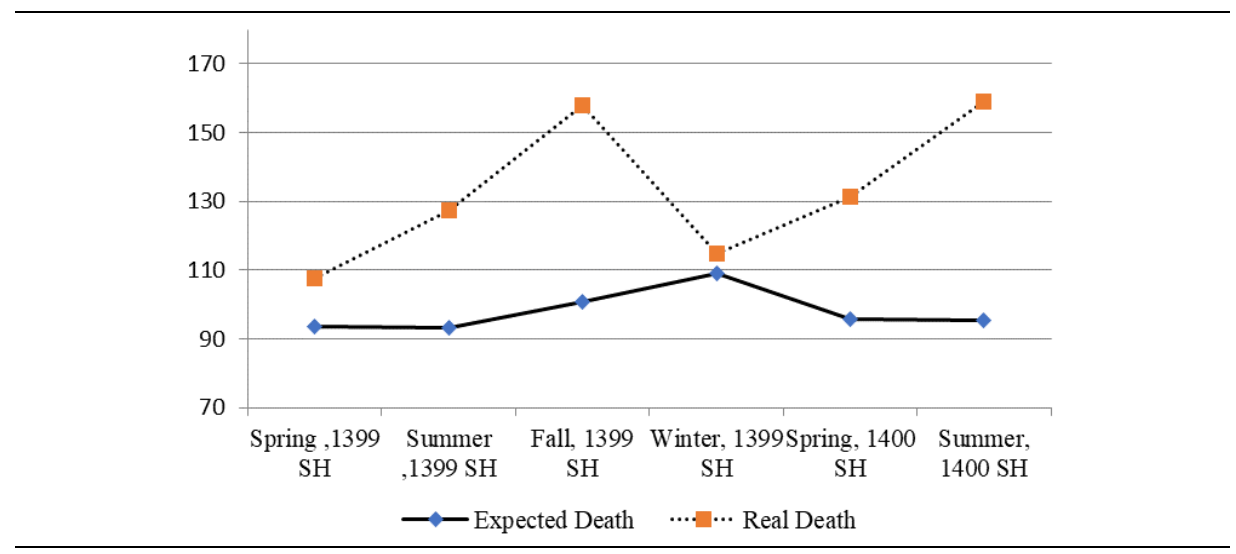

Figure 2. The report of the trend of real death in comparison with the expected death related to the COVID-19 pandemic 


\section{Discussion}

The study of the overall effect of the COVID-19 pandemic on national mortality rate is a crucial methods in estimating the impairment caused by this disease. However, the lack of different types of death coding, false negative of diagnostic tests, and the inability of health systems to perform widespread COVID-19 tests tend to offset the COVID-19 pandemic mortality analysis $(13,14)$. One way to overcome this challenge is to use EMR index .

This index estimates the amount of deaths added in the community based on the records provided by the NOCR. Due to the simplicity and availability of data need to apply in this index, it can be used for calculation in all countries and it can provide an accurate estimate of the COVID-19-related mortalities.

The results of this study showed that the EMR of Iran in COVID-19 pandemic was 36\% (Male $=35 \%$, Female=36). The EMR reported in this study is lower than the EMRs reported by Freitas et al. (15) and Strang et al. (16) and higher than those reported by Michelozzi et al. (17), Woolf et al. (18), Weinberger et al. (19), and Vieira et al .[20]

In particular, Strang et al. (16) showed EMR of 23\% and 113\% in Sudan, in March and April 2020, respectively. Moreover, Freitas et al. (15) reported EMR of $66 \%$ in six Brazilian (COVID-19 period up until May 2, 2020), Michelozzi et al. (17) reported EMR of 19\% in Italian cities (COVID-19 period starts on February 29, 2020), and Woolf et al. (18) demonstrated EMR of $20.8 \%$ in fifty US states and the District of Columbia (January to April 2020). Furthermore, Freitas et al. (15) reported EMR of $66 \%$ in six Brazilian capitals (COVID-19 period until May 2, 2020), Weinberger et al. (19) showed EMR of $18.5 \%$ in the United States (March to May, 2020), and Vieira et al. (20) showed EMR of $15.8 \%$ in Portugal (March to May, 2020).

Furthermore, the results of this study revealed that the provinces of Alborz and Qazvin had the highest EMR indices compared to the other provinces. This finding can be justified by the fact that these two provinces are the passage to reach to the Northern provinces, the main recreational areas in Iran, especially for the citizens of Tehran, the capital of Iran. Therefore, the high EMR index might be due to the passengers transport among these areas. The evidences also have introduced travel as a major risk factor for spreading of SARSCoV-2 (21-23).

Our findings also revealed that the provinces of the southern strip of Iran, which are generally nonindustrial, have a lower EMR index than other provinces. The reason for this might be due to air pollution and also the low movement of the habitants of these provinces with Qom and Tehran (the first provinces that reported COVID-19 in Iran). Previous studies have reported a negative correlation between air quality and COVID-19related death (24-26).

Our study also demonstrated that the provinces of Khuzestan and Bushehr had high EMR indices compared to the other provinces. I can be justified by the fact that these two provinces are the southeast provinces of Iran and have low quality health system. Another reason might be the high rate of travels from the Arab countries of southern border of Persian Gulf to these provinces.

The limitation of this study was lack of access to the reports of MoHME on COVID-19 mortality rates by province. Hence, there are several strong points in this study that should be considered, including applying the complex and powerful statistical methods for mortality rate estimation and the geographical presentation of the EMR by a GIS method.

\section{Conclusion}

Due to the diversity of EMR in different provinces of Iran, the type of management of the provinces with low and moderate EMR can be deliberated as a suitable model to control EMR in provinces with high and very high EMR.

\section{Acknowledgments}

We gratefully acknowledge the financial support provided by Tarbiat Modares University.

\section{Footnotes}

Conflicts of Interest: The authors declare no conflict of interests.

\section{References}

1. WHO characterizes COVID-19 as a pandemic WHO.Available at: URL:https://www.who.int/emergencies/diseases/novelcoronavirus-2019/events-as-they-happen. 2020.

2. WHO. Coronavirus disease (COVID-19) pandemic Available at: https://www.who.int/emergencies/diseases/novelcoronavirus-2019?gclid=CjwKCAiA8Jf-BRBEiwAWDtEGnT5aEuG4ks8f8z7dtHGUcjaM39WLnJ7x3mIhCI7q pdwCot2ITMuOxoCrgkQAvD_BwE. 2020.

3. Tadbiri H, Moradi-Lakeh M, Naghavi M. All-cause excess mortality and COVID-19-related deaths in Iran. Med J Islam Repub Iran. 2020;34:1-6. doi: 10.34171/mjiri.34.80. [Pub Med: 33306040].4.

4. Goldstein ND, Burstyn I. On the importance of early testing even when imperfect in a pandemic such as COVID-19. Glob Epidemiol. 2020;2(323):1-12. doi: 10.1016/j.gloepi.2020.100031. [Pub Med: 32995744]

5. Kandel N, Chungong S, Omaar A, Xing J. Health security capacities in the context of COVID-19 outbreak: an analysis of International Health Regulations annual report data from 182 countries. Lancet. 2020;395(10229):1047-53. doi: 10.1016/S01406736(20)30553-5.

6. Zainol Rashid Z, Othman SN, Abdul Samat MN, Ali UK, Wong KK. Diagnostic performance of COVID-19 serology assays. Malays J Pathol. 2020;42(1):13-21. [Pub Med: 32342927].

7. National Organization for Civil Registration (NOCR). Registered Deaths by Province by Sex. Tehran, Iran: 2020 Available from: 
https://www.sabteahval.ir/avej/Page.aspx?mId=49826\&ID=218 2\&Page=Magazines/SquareshowMagazine; 2020.

8. Leon DA, Shkolnikov VM, Smeeth L, Magnus P, Pechholdová M, Jarvis CI, et al. COVID-19: a need for real-time monitoring of weekly excess deaths. Lancet. 2020;395(10234):e81. doi: 10.1016/S0140-6736(20)30933-8. [Pub Med: 32333839].

9. Fesharaki MG, Kazemnejad A, Zayeri F, Sanati J, Akbari H. Historical cohort study on the factors affecting blood pressure in workers of polyacryl iran corporation using bayesian multilevel modeling with skew $\mathrm{T}$ distribution. Iran Red Crescent Med J. 2013;15(5):418-23. doi: 10.5812/ircmj.10930. [Pub Med: 24349731]

10. Snijders TA, Bosker RJ. Multilevel analysis: An introduction to basic and advanced multilevel modeling: Sage. 1999.

11. Goldstein H. Handbook of Multilevel Analysis. New York: Springer. 2008.

12. COLOR MAPs with STATISTICAL DATA. 2021 [Available from: https://paintmaps.com/map-charts/105/Iran-map-chart.

13. Polyakova M, Kocks G, Udalova V, Finkelstein A. Initial economic damage from the COVID-19 pandemic in the United States is more widespread across ages and geographies than initial mortality impacts. Proc Natl Acad Sci U S A. 2020;117(45):27934-9. doi: 10.1073/pnas.2014279117.

14. Blangiardo M, Cameletti M, Pirani M, Corsetti G, Battaglini M, Baio G, et al. Estimating weekly excess mortality at subnational level in Italy during the COVID-19 pandemic. PloS one. 2020;15(10):1-15. doi: :10.1371/journal.pone.0240286. [Pub Med: 33035253].

15. Freitas ARR, Medeiros NMd, Frutuoso LCV, Beckedorff OA, Martin LMAd, Coelho MMdM, et al. Tracking excess deaths associated with the COVID-19 epidemic as an epidemiological surveillance strategy-preliminary results of the evaluation of six Brazilian capitals. Rev Soc Bras Med Trop. 2020;53:1-8. doi: 10.1590/0037-8682-0558-2020.

16. Strang P, Fürst P, Schultz T. Excess deaths from COVID-19 correlate with age and socio-economic status. A database study in the Stockholm region. Ups J Med Sci. 2020;125(4):297-304. doi: 10.1080/03009734.2020.1828513 . [Pub Med:33100083].

17. Michelozzi P, de'Donato F, Scortichini M, Pezzotti P, Stafoggia $M$, De Sario $M$, et al. Temporal dynamics in total excess mortality and COVID-19 deaths in Italian cities. BMC Public
Health. 2020;20(1):1-8.

18. Woolf SH, Chapman DA, Sabo RT, Weinberger DM, Hill L. Excess Deaths From COVID-19 and Other Causes, March-April 2020 JAMA. 2020;324(5):510-3. doi: 10.1001/jama.2020.11787. [Pub Med: 32609307].

19. Weinberger DM, Chen J, Cohen T, Crawford FW, Mostashari F, Olson D, et al. Estimation of Excess Deaths Associated With the COVID-19 Pandemic in the United States, March to May 2020. JAMA Intern Med. 2020;180(10):1336-44. doi: 10.1001/jamainternmed.2020.3391. [Pub Med: 32609310].

20. Vieira A, Peixoto VR, Aguiar P, Abrantes A. Rapid Estimation of Excess Mortality during the COVID-19 Pandemic in Portugal Beyond Reported Deaths. J Epidemiol Glob Health. 2020;10(3):209-13. doi: 10.20344/amp.13928. [Pub Med: 32343650].

21. Chinazzi M, Davis JT, Ajelli M, Gioannini C, Litvinova M, Merler $S$, et al. The effect of travel restrictions on the spread of the 2019 novel coronavirus (COVID-19) outbreak. Science. 2020;368(6489):395-400. doi: 10.1126/science.aba9757. [PubMed: 32144116].

22. Gostic K, Gomez AC, Mummah RO, Kucharski AJ, Lloyd-Smith JO. Estimated effectiveness of symptom and risk screening to prevent the spread of COVID-19. Elife. 2020;9:1-18. doi: 10.7554/eLife.55570. [Pub Med: 32091395].

23. Nicola M, Alsafi Z, Sohrabi C, Kerwan A, Al-Jabir A, Iosifidis C, et al. The socio-economic implications of the coronavirus pandemic (COVID-19): A review. Int J Surg. 2020;78:185-93. doi: 10.1016/j.ijsu.2020.04.018. [Pub Med: 32305533].

24. Bashir MF, Ma B, Bilal, Komal B, Bashir MA, Tan D, et al. Correlation between climate indicators and COVID-19 pandemic in New York, USA. Sci Total Environ. 2020;728:1-5. doi: 10.1016/j.scitotenv.2020.138835. [Pub Med: 32334162].

25. Li H, Xu XL, Dai DW, Huang ZY, Ma Z, Guan YJ, et al. Air pollution and temperature are associated with increased COVID-19 incidence: A time series study. Int J Infect Dis. 2020;97:278-82. doi: 10.1016/i.ijid.2020.05.076. [Pub Med: 32502664].

26. Zhang X, Tang M, Guo F, Wei F, Yu Z, Gao K, et al. Associations between air pollution and COVID-19 epidemic during quarantine period in China. Environ Pollut. 2021;268(Pt A):1-

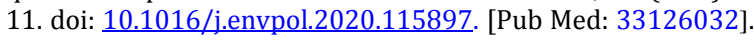

